\title{
A position control method for vertical rotor orientation system
}

\author{
Sijia Liu ${ }^{\mathrm{a}}$, Yu Fan, Jun Di and Mingming Ji \\ School of Electrical Engineering, Beijing Jiaotong University, Beijing, 100044, China
}

\begin{abstract}
Vertical rotating devices may cause large amplitude vibration or even lose stability during low-speed operation. This paper discusses the control approaches of a low-speed vertical rotor orientation system based on the electromagnetic levitation. In the paper, according to the gyro mechanics principle, the nonlinear dynamic equations of the vertical rotor system are established and the kinetic characteristics of the system in the gravitational field are analyzed. Considering the uncertainties of the system, a position control strategy used for the vertical rotor orientation system is presented based on the gyroscope effect. Finally, the simulation of the control system and experiment system are carried out and set up to verify the feasibility and effectiveness of the control method. Results show that the presented control approach is able to achieve a stable positioning control for the spinning rotor.
\end{abstract}

Keywords: magnetic bearing; gyroscope effect; uncertainty; nonlinear; position control.

\section{Introduction}

The vertical rotor devices are widely applied in civilian and industrial fields, such as washing machines and centrifuges. These devices, due to their functional requirement, are often without mechanical bearing on the top-side of the rotor. The one-side bearing structure is hard to control the vibrations and noises caused by the mass eccentricity, and may also aggravate the abrasion of devices. A traditional method to solve this problem is to use a hydraulic balancer[1-3]to reduce the vibration. However, although has advantages such as simple and easy to be applied, the method is a passive way to restrain the vibration. The major disadvantages of the passive control method are 1)unable to eliminate the vibration entirely; 2) easier affected by the load variation and under some circumstance evenfailure.

Therefore, an active vibration reduction system, or namely rotor orientation system may provide a better solution of this problem. The active orientation system can be achieved via the active magnetic bearing(AMB) system.

Similarity of the washing machine and the AMB is that they both contain a spinning rotor, which can be regarded as a gyro and follows the gyroscope effect. Nevertheless, most researches on AMB focus on a high speedstate(often over $10000 \mathrm{rpm}$ [4-7]). Under lower angularspeed, however, the gyroscope effect will become weak and may fail to sustain a stable whirling. The vibration of the rotor will be the mixture of the gyroscope effect and other uncertainties. Furthermore, when lying vertically,

acorresponding author : 116s38j31@hotmail.com

(C) 2016. The authors - Published by Atlantis Press 
the rotor will behave a nonlinear dynamic performance[8]. On this occasion, the control methods based on the high speed may not be feasible. A specific control approach is necessary.

Under lower speed, the gyroscope effect, although rather weak, still affects the rotor as long as the rotor is spinning. This effect points out that, instead of moving towards the external force, the rotor will move towards the direction of the external torque. If this feature still can be utilized in design of the control system, the control may be largely simplified.

Therefore, in the paper, Section 2 describes the theorem of angular momentum and establishes the mathematical models of a one gimbal vertical rotor AMB system, using the Cardan Angles[9]as system state variables. Section 3 analyses the dynamic features of the vertical rotor system. Based on gyroscope effect, a feedback position control method is proposed. Section 4 shows the simulation results of the presented control method. Finally, an experiment test is established to verify the effectiveness of the method and the testing results are given in Section 5.

\section{Modeling of the vertical AMB system}

\subsection{Description of the rotor attitude}

A sketch diagram of the rotor this paper discusses is shown inFigure1. The rotor is fixed at point $O$ by a gimbal, so there is no translational motion. $O_{C}$ is the centroid of the rotor. Neglecting the deformation of the rotor, it is a symmetrical rigid-body about its axis $\mathrm{OO}_{C}$. The motion of the rotor can be decomposed into two parts: the first is the rotation about its axis; the second is the swinging of the rotor axis about the vertical direction. In order to describe the rotor attitude and the complex rotation accurately, a static coordinate $O X Y Z$ and a Resal's coordinate $O x_{1} y_{1} z_{1}$ need to be established. Ignoring the rotation of the earth, $O X Y Z$ can be treated as an inertial coordinate. The Resal's frame always moves with the rotor, but do not rotate. The position of the rotor in this coordinate is static. The rotor only spins about the $O z_{1}$ axis. Therefore, the attitude of the rotor in the static coordinate can be expressed as the transformation of coordinates $O X Y Z$ and $O x_{1} y_{1} z_{1}$.

This transformation, as shown inFigure1, includes two steps: first, rotate $O X Y Z$ about $O X$ axis through $\alpha$, to obtain the coordinate $O x_{0} y_{0} z_{0}$; then rotate $O x_{0} y_{0} z_{0}$ about $O y_{0}$ axis through $\beta$, to obtain the Resal's coordinate $O x_{1} y_{1} z_{1}$. If continuing rotating the $O x_{1} y_{1} z_{1}$ about $O z_{1}$ through $\gamma$ to get a coordinate naming $\mathrm{Ox}_{2} y_{2} z_{2}$, these angles $\alpha, \beta, \gamma$ are the Cardan angles, and this coordinate $\mathrm{Ox}_{2} y_{2} z_{2}$ is a body-fixed coordinate system. However, in this paper, because of the symmetry of the rotor, the dynamic equations will be simplified in the Resal's coordinate. Thus the following discussion will be confined to it. The angle $\theta$ is the angular displacement of $O z_{1}$ from $O Z$, and also the displacement of the rotor axis.

The transition matrix from the static to the Resal's coordinate is

$$
\mathbf{T}_{X-x_{1}}=\mathbf{T}_{x_{0}-x_{1}} \mathbf{T}_{X-x_{0}}=\left[\begin{array}{ccc}
\cos \beta & \sin \alpha \sin \beta & -\cos \alpha \sin \beta \\
0 & \cos \alpha & \sin \alpha \\
\sin \beta & -\sin \alpha \cos \beta & \cos \alpha \cos \beta
\end{array}\right]
$$

and inverse Eq.(1)to get the transition matrix from the Resal's to static the coordinate

$$
\mathbf{T}_{x_{1}-X}=\mathbf{T}_{X-x_{1}}^{-1}=\left[\begin{array}{ccc}
\cos \beta & 0 & \sin \beta \\
\sin \alpha \sin \beta & \cos \alpha & -\sin \alpha \cos \beta \\
\cos \alpha \sin \beta & \sin \alpha & \cos \alpha \cos \beta
\end{array}\right]
$$


In the Resal's frame, as $\mathrm{Oz}_{1}$ always coincides the principal axis of the rotor, the coordinates of the rotor centroid $O C$ can be assumed as $\xi_{1}=\left(\begin{array}{lll}0 & 0 & c\end{array}\right)^{\circ}$. Then in $O X Y Z$, the coordinates of the centroid is

$$
\xi=\mathbf{T}_{x_{1}-X} \xi_{1}=c(\sin \beta \quad-\sin \alpha \cos \beta \quad \cos \alpha \cos \beta)^{\bullet}
$$

According to Eq. (2)the attitude of the rotor can be described by the centroid's coordinates.

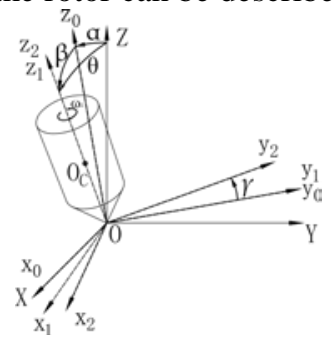

Figure 1. Schematic diagram of rigid body's fixed point rotation inCardan’s angle.

\subsection{Kinematical equations of the rotor}

As mentioned above, $\mathrm{Oz}_{1}$ overlaps with the rotor axis all the time. Therefore, the rotor motion to static coordinates is a synthesis two motions. The first is the relative motion of the rotor in the Resal's frame $O x_{1} y_{1} z_{1}$ about its own principal axis (i.e. $O z_{1}$ ), naming this angular speed be $\omega_{z}$. The second is the transport motion of the Resal's frame $O x_{1} y_{1} z_{1}$ in the static frame $O X Y Z$, naming this angular speed vector be $\omega_{1}$.

It is obvious that the rotation speed of the rotor about its axis is

$$
\dot{\omega}_{z}=\gamma
$$

Next, according to the generation process of theCardan's frame we can yield that the angular speed $\omega_{1}$ can be expressed as

$$
\omega_{1}=\dot{\alpha} \mathbf{i}_{X}+\dot{\beta} \mathbf{j}_{0}
$$

where $\mathbf{i}_{X}, \mathbf{j}_{0}$ are the base vectors of angular speed of $O X$ and $O y_{0}$ axis respectively.

Eq.(3)can be rewritten in the scalar components form as

$$
\left\{\begin{array}{l}
\omega_{1 x}=\dot{\alpha} \cos \beta \\
\omega_{1 y}=\dot{\beta} \\
\omega_{1 z}=\dot{\alpha} \sin \beta
\end{array}\right.
$$

As $O z_{1}$ axis always coincides with the rotor axis but does not participate the spinning, the components of the rotor angular speeds satisfy

$$
\omega_{x}=\omega_{1 x}, \omega_{y}=\omega_{1 y}
$$

but $\omega_{z} \neq \omega_{1 z}$.

Combining the equations above, the kinematical formula of the rotor is 


$$
\left\{\begin{array}{l}
\dot{\alpha}=\omega_{x} / \cos \beta \\
\dot{\beta}=\omega_{y} \\
\dot{\gamma}=\omega_{z}
\end{array}\right.
$$

\subsection{Dynamic equations of the rotor}

Rigid-body's motion obeys the theorem of angular momentum,

$$
\frac{\mathrm{d}}{\mathrm{d} t} \boldsymbol{L}_{O}=\boldsymbol{M}_{O}
$$

and in the Resal's coordinate, the dynamic equation can be expressed as

$$
\left\{\begin{array}{l}
\dot{L}_{x}+\left(\omega_{1 x} L_{z}-\omega_{1 z} L_{y}\right)=M_{x} \\
\dot{L}_{y}+\left(\omega_{1 z} L_{x}-\omega_{1 x} L_{z}\right)=M_{y} \\
\dot{L}_{z}+\left(\omega_{1 x} L_{y}-\omega_{1 y} L_{x}\right)=M_{z}
\end{array}\right.
$$

Considering the momentum equation $L_{x}=J_{x} \omega_{x}, L_{y}=J_{y} \omega_{y}, L_{z}=J_{z} \omega_{z}$ and the symmetry of the rotor, Eq. (8)can be written as

$$
\left\{\begin{aligned}
J_{x y} \dot{\omega}_{x}+\left(J_{z} \omega_{z}-J_{x y} \omega_{1 z}\right) \omega_{y} & =M_{x} \\
J_{x y} \dot{\omega}_{y}+\left(J_{x y} \omega_{1 z}-J_{z} \omega_{z}\right) \omega_{x} & =M_{y} \\
J_{z} \dot{\omega}_{z} & =M_{z}
\end{aligned}\right.
$$

Eq.(9)is the dynamic equation of the rotor, where $J_{x y} \triangleq J_{x}=J_{y}$.

\subsection{System state equations}

In Eq.(9), the external torques on the right side includes both the gravitational moment and the control torque. First we analyze the free situation that only gravity exists.

The gravitational moment on the rotor can be expressed as

$$
\boldsymbol{M}_{O}=\overrightarrow{O O}_{C} \times \boldsymbol{P}
$$

In the static coordinate $O X Y Z$, gravity $\boldsymbol{P}=P \mathbf{k}$, where $\mathbf{k}$ is the base vector of $O Z$. Assuming $O O_{C}=c$, then in the Resal's coordinate, $\overrightarrow{O O}_{C}=c \mathbf{k}_{1}$, where $\mathbf{k}_{1}$ is the base vector of $O z_{1}$. Using Eq. (1)we can transform $\overrightarrow{\mathrm{OO}}_{C}$ into the static coordinate, i.e.

$$
\overrightarrow{O O}_{C}=\mathbf{T}_{x_{1}-X}\left[\begin{array}{lll}
0 & 0 & c
\end{array}\right]^{\text {㖺 }}=c\left[\begin{array}{lll}
\sin \beta & -\sin \alpha \cos \beta & \cos \alpha \cos \beta
\end{array}\right]
$$

Substitute Eq. (11)and the gravity equation into Eq. (10)

$$
\left\{\begin{array}{l}
M_{x}=P c \sin \alpha \cos \beta \\
M_{y}=P c \sin \beta \\
M_{z}=0
\end{array}\right.
$$

If there exists other external moments, Eq. (12)can be transformed into 


$$
\left\{\begin{array}{l}
M_{x}=P c \sin \alpha \cos \beta+M_{y e} \\
M_{y}=P c \sin \beta+M_{x e} \\
M_{z}=M_{z e}
\end{array}\right.
$$

Substitute Eq. (13)into Eq.(9), and unite with Eq.(6). Let $x_{1}=\alpha, x_{2}=\beta, x_{3}=\omega_{x}, x_{4}=\omega_{y}$, $x_{5}=\omega_{z}$, we can obtain the states equations of the system

$$
\left\{\begin{array}{l}
\dot{x}_{1}=\frac{x_{4}}{\cos x_{2}} \\
\dot{x}_{2}=x_{5} \\
\dot{x}_{3}=x_{6} \\
\dot{x}_{4}=\frac{P c}{J_{x y}} \sin x_{1} \cos x_{2}-\frac{J_{z}}{J_{x y}} X_{5} x_{6}+x_{4} x_{5} \tan x_{2}+\frac{M_{x c}}{J_{x y}} \\
\dot{x}_{5}=\frac{P C}{J_{x y}} \sin x_{2}-x_{4}^{2} \tan x_{2}+\frac{J_{z}}{J_{x y}} x_{4} x_{6}+\frac{M_{y c}}{J_{x y}} \\
\dot{x}_{6}=\frac{M_{z c}}{J_{z}}
\end{array}\right.
$$

where $M_{x c}, M_{y c}, M_{z c}$ are the external control torques.

\section{Position control method design}

\subsection{Dynamic analysis of the system}

System determined by Eq. (14)is a set of five-order nonlinear coupling equations; direct analysis is difficult. However, noticing that the 5th sub-equation is decoupled from the rest, without loss of generality, we assume that the state component $x_{5}$ is a constant. Hence, Eq. (14)can be reduced to a four-order differential equation. Obviously, origin is equilibrium of the system. Linearly expand system equation at equilibrium and obtain the Jacobi matrix

$$
\mathbf{J}=\left[\begin{array}{cccc}
0 & 0 & 1 & 0 \\
0 & 0 & 0 & 1 \\
\frac{M_{0}}{J_{x y}} & 0 & 0 & -\frac{L}{J_{x y}} \\
0 & \frac{M_{0}}{J_{x y}} & \frac{L}{J_{x y}} & 0
\end{array}\right]
$$

Where $L=J_{z} x_{5}, M_{0}=P c$.

According to the Routh-Hurwitzcriterion, it is obvious that $\mathbf{J}$ has eigenvalues on the right half plane when $L^{2}<4 M_{0} J_{x y}$. That is to say, under a lower rotating speed, the gyroscope effect may be insufficient to maintain a stable precession of the rotor. Thus, the rotor may deflect from the vertical position arbitrarily, and many environmental factors may affect the rotor vibration.

\subsection{Position control method}

Although the gyroscope effect may not enough to sustain a stable rotation, the rotor will still be under this rule. As mentioned above, the spinning rotor will move towards the external moment. Therefore if the control torque points to center all the time, there will be a moving trend of the rotor to the vertical position. Choosing a certain control torque may obtain a stable motion of the rotor. 
In order to get the control torque, first projecting Figure2to the $O X Y$ plane, and substituting Eq.(11), we can obtain the conceptual graph of the control torque as shown in Figure2.

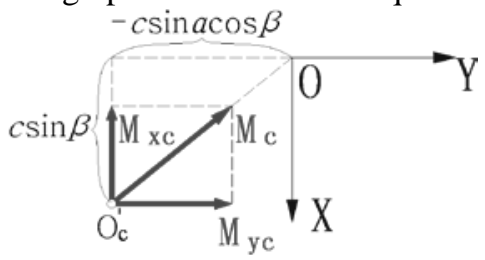

Figure2. Conceptual graph of control torque.

A proper control torque pointing to the center all the time can be $\boldsymbol{M}_{\mathrm{c}}=C \boldsymbol{d}$, where $\boldsymbol{d}={\overrightarrow{O O^{\prime}}}_{\mathrm{C}}, \mathrm{C}$ is proportional to the mass of the rotor, and $O^{\prime}{ }_{C}$ is the projection of centroid $O_{C}$ to the $O X Y$ plane. It is obvious that the length of ${\overrightarrow{O O^{\prime}}}_{C}$ is $d=c \sqrt{\sin ^{2} \beta+\sin ^{2} \alpha \cos ^{2} \beta}$, and the scalar expressions of control torque $\boldsymbol{M}_{\mathrm{c}}$ is

$$
\left\{\begin{array}{l}
M_{x 1}=-C \sin \beta \\
M_{y 1}=C \sin \alpha \sin \beta
\end{array}\right.
$$

When $\alpha$ and $\beta$ are both small angles, Eq. (15)can be approximately rewritten as

$$
\left\{\begin{array}{l}
M_{x 1}=-C \beta \\
M_{y 1}=C \alpha
\end{array}\right.
$$

and on this occasion, the angular displacement of the rotor axis from $O Z$ can also be approximately expressed as

$$
\theta=\sqrt{\alpha^{2}+\beta^{2}}
$$

To ensure the stability the system, the control torques need to be contain damping components. A feasible damping is

$$
\left\{\begin{array}{l}
M_{x 2}=-K \dot{\alpha} \\
M_{y 2}=-K \dot{\beta}
\end{array}\right.
$$

Then the total control torque is

$$
\left[\begin{array}{l}
M_{x c} \\
M_{y c}
\end{array}\right]=\left[\begin{array}{l}
M_{x 1}+M_{x 2} \\
M_{y 1}+M_{y 2}
\end{array}\right]=\left[\begin{array}{c}
-C x_{2}-K x_{3} \\
C x_{1}-K x_{4}
\end{array}\right]
$$

Substituting Eq.(18)into Eq.(14), yields the Jacobi matrix of the system is

$$
\mathbf{J}_{2}=\left[\begin{array}{cccc}
0 & 0 & 1 & 0 \\
0 & 0 & 0 & 1 \\
\frac{M_{0}}{J_{x y}} & -\frac{C}{J_{x y}} & -\frac{K}{J_{x y}} & -\frac{L}{J_{x y}} \\
\frac{C}{J_{x y}} & \frac{M_{0}}{J_{x y}} & \frac{L}{J_{x y}} & -\frac{K}{J_{x y}}
\end{array}\right]
$$

Its characteristic polynomial is 


$$
\lambda_{2}(s)=s^{4}+\frac{2 K}{J_{x y}} s^{3}+\left(\frac{J^{2}+K^{2}}{J_{x y}^{2}}-\frac{2 M_{0}}{J_{x y}}\right) s^{2}+\frac{2\left(C L-K M_{0}\right)}{J_{x y}^{2}} s+\frac{C^{2}+M_{0}^{2}}{J_{x y}^{2}}
$$

According to Routh-Hurwitz criterion, when $C, K$ satisfy the following inequalities, all the roots of $\lambda_{2}(s)$ have positive parts and locate on the left half plane:

$$
\left\{\begin{array}{l}
K>0 \\
K^{3}+\left(L^{2}-M_{0} J_{x y}\right) K-L J_{x y} C>0 \\
J_{x y} C^{2}-L K C+M_{0} K^{2}<0
\end{array}\right.
$$

Eq.(19)guarantees the stability of the linearized system, thereby insures the stability of the original nonlinear systemin a neighborhood of the equilibrium. Here the feedback factors $C, K$ are free parameters that are only restricted by physical limits, therefore one can always find a proper set of $C, K$ to satisfy Eq. (19)and make the system stable.

\section{Simulation}

\subsection{Simulation of the rotor orientation system}

The mechanical parameters of the rotor are as listed in Table 1.According to the parameters in Table 1, the angular momentums of the rotor are $J_{x y}=0.00348 \mathrm{~kg} \cdot \mathrm{m}^{2}, J_{z}=0.0139 \mathrm{~kg} \cdot \mathrm{m}^{2}$. Substitute these parameters and Eq.(18)into Eq.(14), we will obtain the projection diagram of system trajectory on the $x_{1}-x_{2}$ plane is shown as in Figure3 from different initial $\alpha$ and $\beta$, which shows the trails of the rotor axis from different initial attitudes.

Table 1. Parameters of the rotor

\begin{tabular}{ccc}
\hline Parameter name & Value & Units \\
\hline Rotor mass & 2.797 & $\mathrm{~kg}$ \\
Outer diameter & 150 & $\mathrm{~mm}$ \\
Inner diameter & 142 & $\mathrm{~mm}$ \\
Rotor height & 150 & $\mathrm{~mm}$ \\
Rotor material & Iron & -- \\
\hline
\end{tabular}

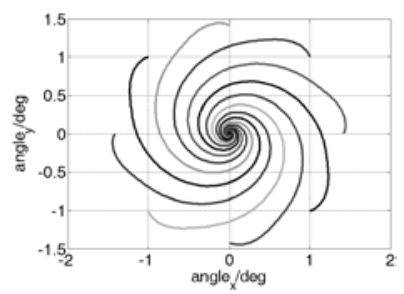

Figure 3. Rotor displacement from different initials

From Figure3we can see that after the feedback of Eq.(18), the rotor axis will asymptotically approach to the vertical direction.Figure4illustrates one of the time response of the angular displacement $\theta$. The orientation control system starts at $7.5 \mathrm{~s}$. It also shows that $\theta$ will reduce asymptotically to zero after the control system effects. 


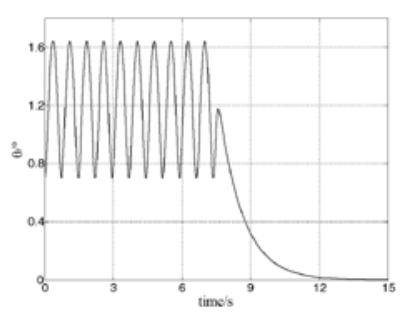

Figure 4.Angular displacement of the rotor without disturbance

\subsection{Simulation in view of uncertainties}

The simulation results illustrated in Figure3 and Figure4neglecting the actual disturbances (e.g. the collision between the rotor and protection bearing) and additional modules (e.g. filters and power amplifiers). When considering these factors, system (14)will no longer be continuous, and the actual response of the rotor drift will be much more complicated. On this occasion, however, simulation results show that the orientation approach in Eq.(18) is still effective.

Figure5shows the time response of the angle $\theta$ when following uncertainties exist:

1. The collision between the rotor and the protection bearing.

2. A small amplitude of sinusoidal interference signal with the frequency of the rotor spinning $\omega_{z}$, which reflects the affects from the rotor self-rotation.

It can be concluded from Figure5 that although disturbances existed, the rotor will still approach the vertical direction $\mathrm{OZ}$ asymptotically.

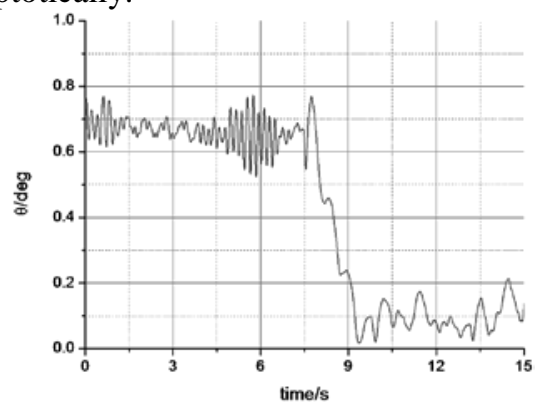

Figure 5. Time response of $\theta$ considering disturbances

\section{Experiment results of the control system}

\subsection{Experimental apparatus}

A photo of the experimental table is shown in Figure6(a).Its side-view is shown in Figure6(b). The four electromagnets locate perpendicularly around the rotor as the actuator of the orientation forces. The iron rotor in the center is connected to a motor beneath with a universal joint, and the top side of the rotor is free. The rotor can rotate with the motor while its axis can drift within a certain angle. The two gap sensors are installed in $90^{\circ}$ interval and provide the control signals to the controller. 

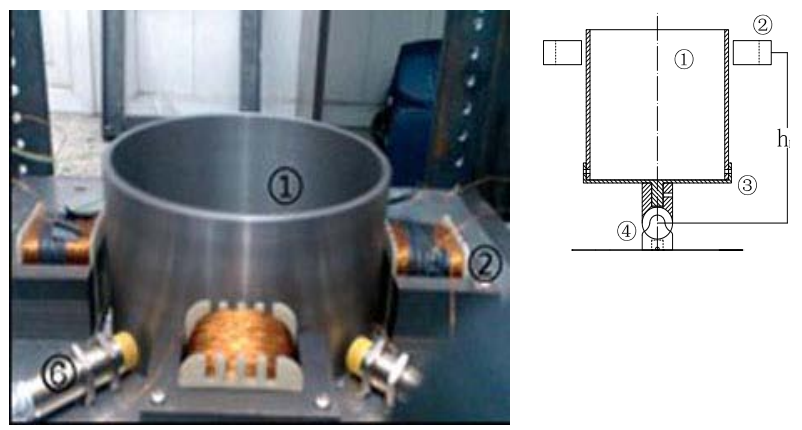

(a) Photo of the experiment platform $\quad$ (b) Rotor system design

Figure 6. Photo of the experimental table.1. Rotor;2. electromagnet; 3. end cap;4. universal bearing; 5. motor; 6.gap sensor

\subsection{Experiment program}

The block diagram of the experiment is shown in Figure7. The whole control process (expect the power amplification) is established using dSPACE. dSPACE is a high performance semi-physical simulation instrument. It integrates signal acquisition, computation, and D/A conversion blocks and can complete these procedure just according to the control model established in Matlab/Simulink. It also has a convenient communication interface with computer to achieve the storage, handle, and display of the experimental results. This tool simplifies the implementation of the controller and enables the authors to focus on the feasibility of the control method.

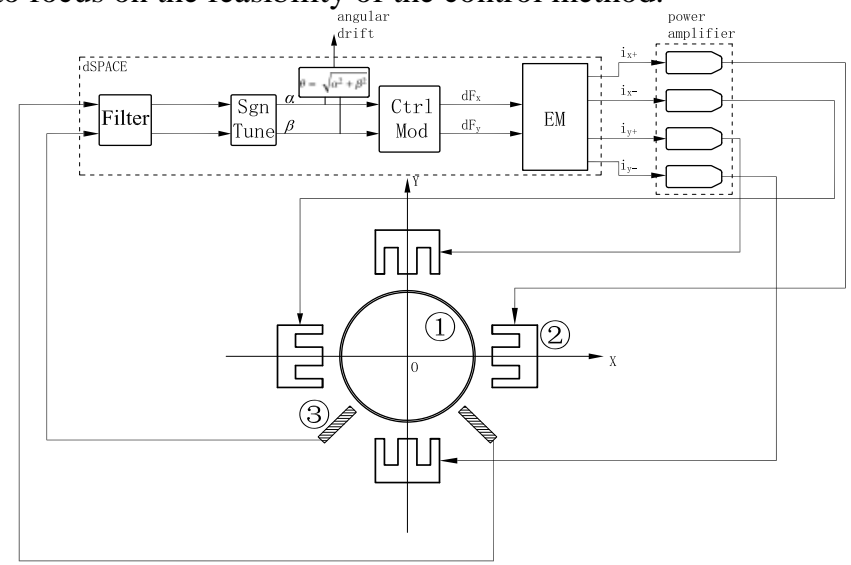

Figure 7. Block diagram of the experiment.1.rotor; 2.electromagnet;3.gap sensor.

The experiment program is as following:

1. Measurement of the rotor drift angle

The drift angles of the rotor in $X / Y$ direction angle $_{x}$ and angle ${ }_{y}$ are converted from the two gap values gap $_{1}$ and $\operatorname{gap}_{2}$. The two angles are the state variables $x_{1}$ and $x_{2}$.

2. Calculation of the electromagnetic force

Substitute angle $_{x}$, angle ${ }_{y}$, and their derivatives into Eq.(18), the control torque $M_{x c}$ and $M_{y c}$ can be calculated, and the control forces to the rotor in $X / Y$ direction are

$$
\left\{\begin{array}{l}
F_{x c}=M_{x c} / h_{m} \\
F_{y c}=M_{y c} / h_{m}
\end{array}\right.
$$


In order to make the control force continuous and smooth, the force $\mathrm{d} F_{x}$ and $\mathrm{d} F_{y}$ are realized via differential control of a pair of electromagnets, i.e., when $\mathrm{d} F_{x}=\mathrm{d} F_{y}=0$, each of the four electromagnets has a reference force $F_{0}$. Therefore the force of each electromagnet is

$$
\left\{\begin{array}{l}
F_{x+}=F_{0}+\frac{F_{x c}}{2} \\
F_{x-}=F_{0}-\frac{F_{x c}}{2} \\
F_{y+}=F_{0}+\frac{F_{y c}}{2} \\
F_{y-}=F_{0}-\frac{F_{y c}}{2}
\end{array}\right.
$$

3. Calculation of current in every electromagnet

According to the experimental data, the electro-magnetic force conforms to

$$
F=\frac{a I^{2}}{(b+g)^{2}}
$$

where $a, b$ are factors corresponding to the electromagnet, and $g$ is the gap between the electromagnet and the rotor.

Substitute Eq. (18)and (22) into Eq.(21), the currents of the four electromagnets are

$$
\left\{\begin{array}{l}
I_{x+}=\left(b+g_{x+}\right) \sqrt{\left(F_{\mathrm{n} 0}+\frac{M_{x c}}{2 h_{\mathrm{m}}}\right) / a} \\
I_{x-}=\left(b+g_{x-}\right) \sqrt{\left(F_{\mathrm{n} 0}-\frac{M_{x \mathrm{xc}}}{2 h_{\mathrm{m}}}\right) / a} \\
I_{y+}=\left(b+g_{y+}\right) \sqrt{\left(F_{\mathrm{n} 0}+\frac{M_{y c}}{2 h_{\mathrm{m}}}\right) / a} \\
I_{y-}=\left(b+g_{y-}\right) \sqrt{\left(F_{\mathrm{n} 0}-\frac{M_{y c}}{2 h_{\mathrm{m}}}\right) / a}
\end{array}\right.
$$

Eq.is the control signal of each electromagnet. As discussed above, the factors $C, K$ are free parameters and can be adjusted to achieve the stability of the system.

\subsection{Experiment results}

The mechanical parameters are the same as listed in Table 1. The rotation speed of the rotor is $400 \mathrm{rpm}$. The control system activates at $t=7.5 \mathrm{~s}$. The outputs of the angular drift $\theta$ can be captured and stored in the computer via dSPACE. Then the curves of $\theta$ is shown in Figure8. 


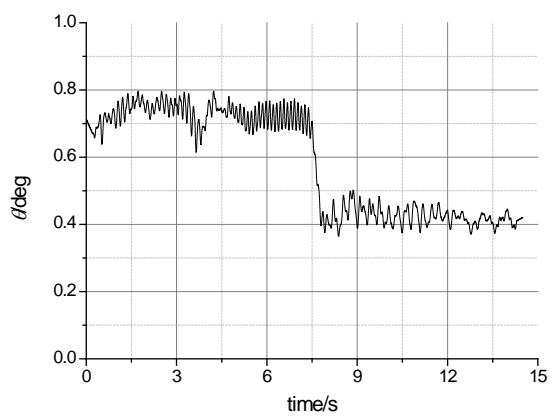

Figure 8. Gap curve of each sensor.

\section{Conclusions}

In the paper, the position control system of a vertical rotor based on theorem of angular momentum and Cardan's angles is established, and the dynamic characteristics of the spinning rotor in the gravity field are analyzed Based on the gyroscope effect, a rotor positioning control method is proposed. Under the situation of nonlinearity and parameter variation, the simulation of the presented method is performed to compare with the traditional pole placement approach. To verify the correctness of the proposed method, an experimental system is set up. The experimental test results show that the position control method can reduce the amplitude of the rotor vibration and maintain the system stability during the rotor rotating effectively.

\section{Acknowledgments}

The work in this paper is supported by National Natural Science Foundation of China (Grant No. 51077003) and the Fundamental Research Funds for the Central Universities (Grant No. 2014YJS131).

\section{References}

1. S. Bae, J. Lee, Y. Kang, J. Kang, and J. Yun, "Dynamic analysis of an automatic washing machine with a hydraulic balancer," Journal of Sound and vibration, vol. 257, no. 1, pp. 3-18, 2002.

2. H.-W. Chen and Q.-J. Zhang, "Stability analyses of a vertical axis automatic washing machine with a hydraulic balancer,” Mechanism and Machine Theory, vol. 46, no. 7, pp. 910 - 926, 2011.

3. H.-W. Chen, Q.-J. Zhang, and S.-Y. Fan, "Study on steady-state response of a vertical axis automatic washing machine with a hydraulic balancer using a new approach and a method for getting a smaller deflection angle,” Journal of Sound and Vibration, vol. 330, no. 9, pp. 20172030, 2011.

4. H. Bleuler, M. Cole, P. Keogh, R. Larsonneur, E. Maslen, R. Nordmann, Y. Okada, G. Schweitzer, and A. Traxler, Magnetic Bearings: Theory, Design, and Application to Rotating Machinery. Berlin Heidelberg: Springer-Verlag, 2009.

5. M. Ahrens, L. KuCera, and R. Larsonneur, "Performance of a magnetically suspended flywheel energy storage device,” IEEE Transactions on Control Systems Technology, vol. 4, no. 5, pp. 494-502, 1996.

6. N. Tsai, C. Kuo, and R. Lee, "Regulation on radial position deviation for vertical amb systems," Mechanical Systems and Signal Processing, vol. 21, no. 7, pp. 2777-2793, 2007. 
7. J. Fang and Y. Ren, "High-precision control for a single-gimbal magnetically suspended control moment gyro based on inverse system method," IEEE Transactions on Industrial Electronics, vol. 58, no. 9, pp. 4331-4342, 2011.

8. M. Shi, D. Wang, and J. Zhang, "Nonlinear dynamic analysis of a vertical rotor-bearing system," Journal of Mechanical Science and Technology, vol. 27, no. 1, pp. 9-19, 2013.

9. W. B. Heard, Rigid body mechanics: mathematics, physics and applications. Weinheim: WileyVCH, 2005. 